\title{
Cultura Cultura
}

Revista de Historia Teorid das ldeaias - Revista de História e Teoria das Ideias

Vol. $34 \mid 2015$

Diderot et la morale

\section{Histoire(s) de caractères}

Le programme étho-poétique des contes

\section{Konstanze Baron}

\section{(2) OpenEdition}

Journals

\section{Édition électronique}

URL : http://journals.openedition.org/cultura/2463

DOI : $10.4000 /$ cultura.2463

ISSN : 2183-2021

Éditeur

Centro de História da Cultura

\section{Édition imprimée}

Date de publication : 9 juin 2015

Pagination : 111-134

ISSN : 0870-4546

\section{Référence électronique}

Konstanze Baron, « Histoire(s) de caractères », Cultura [Online], Vol. 34 | 2015, posto online no dia 12 julho 2016, consultado a 19 abril 2019. URL : http://journals.openedition.org/cultura/2463 ; DOI : $10.4000 /$ cultura. 2463

Ce document a été généré automatiquement le 19 avril 2019

(c) CHAM - Centro de Humanidades / Centre for the Humanities 


\title{
Histoire(s) de caractères
}

\author{
Le programme étho-poétique des contes
}

\author{
Konstanze Baron
}

1 Dans la Réfutation suivie de l'ouvrage d'Helvétius intitulé "L'Homme ", Diderot explique pourquoi il n'a jamais écrit d'éthique, c'est-à-dire de livre philosophique sur la morale :

[s]'il y a des questions en apparence assez compliquées qui m'ont paru simples à l'examen ; il y en a de très simples en apparence que j'ai jugées au-dessus de mes forces. Par exemple, je suis convaincu que, dans une société même assez mal ordonnée que la nôtre, où le vice qui réussit est souvent applaudi, et la vertu qui échoue presque toujours ridicule, je suis convaincu, dis-je, qu'à tout prendre, on n'a rien de mieux à faire pour son bonheur que d'être un homme de bien ; c'est l'ouvrage, à mon gré, le plus important et le plus intéressant à faire ; c'est celui que je me rappellerais avec le plus de satisfaction, dans mes derniers moments ; c'est une question que j'ai méditée cent fois et avec toute la contention d'esprit dont je suis capable ; j'avais, je crois, les données nécessaires. Vous l'avouerais-je ? je n'ai pas même osé prendre la plume pour en écrire la première ligne. Je me disais, Si je ne sors pas victorieux de cette tentative, je deviens l'apologiste de la méchanceté. J'aurai trahi la cause de la vertu. J'aurai encouragé l'homme au vice. Non, je ne me sens pas bastant pour ce sublime travail. J'y consacrerais inutilement toute ma vie.

Bien qu'il soit convaincu, à un niveau tout à fait pratique et personnel, du lien intrinsèque entre le bonheur et la vertu (puisqu' « on n'a rien de mieux à faire pour son bonheur que d'être homme de bien »), Diderot se dit pourtant incapable de prouver l'existence de ce lien de façon théorique. Il renonce donc à écrire cet " ouvrage ", qu'il juge pourtant "le plus intéressant à faire ", de peur d'y échouer au niveau de l'argumentation philosophique, ce qui aurait nécessairement des conséquences néfastes pour la morale. Il vaut mieux se taire, conclut-il, que de se faire, par simple mégarde ou inadvertance, l'avocat du vice.

3 Se présente donc, dans ce passage, un philosophe extrêmement consciencieux consciencieux au point de se condamner lui-même au silence par - quel paradoxe amour de la vertu! Bien entendu, le silence philosophique dont il est question ici n'exclut nullement un autre type d'« ouvrages ", le travail pratique. Laissons-donc la philosophie, et cultivons notre jardin... ? Mais là n'est pas tout. Pour quiconque connaît un peu Diderot, il est clair que sa prétendue « abstinence » en matière de morale n'est pas si 
grande que cela. Au contraire : L'oeuvre de Diderot livre une vaste réflexion sur la morale (une " éthique " donc au sens strict du terme) et qui ne se limite pas à la simple propagation - pratique - de la vertu. Seulement, il faut savoir l'identifier. À ce propos, la suite du passage cité nous donne déjà quelques indications précieuses. Car Diderot poursuit :

Voulez-vous une question plus simple ? La voici. Le philosophe appelé au tribunal des lois, doit-il ou ne doit-il pas avouer ses sentiments, au péril de sa vie. Socrate fit-il bien ou mal de rester dans la prison ? Et combien d'autres questions qui appartiennent plus au caractere qu'à la logique !²

4 C'est seulement en apparence que le philosophe change de sujet, en transformant la question théorique discutée auparavant (" une éthique philosophique est-elle possible ») en une question d'ordre pratique (« le philosophe doit-il...»), et en nous référant, tout de suite, à un cas particulier, spécifié par un nom propre (« Socrate »). Est-ce que les choses deviennent, par ce moyen, plus "simples ", comme il le suggère ? C'est ce dont on pourrait douter. En tout cas, elles deviennent plus plastiques, et plus concrètes. Au lieu de nous perdre dans un débat théorique infructueux, nous sommes invités à nous tourner vers les affaires pratiques de la vie, vers l'agir du philosophe, et à former une opinion à propôs d'un sujet nommé Socrate, qui se trouve placé dans une situation exemplaire et donc, en quelque sorte, paradigmatique. « Fit-il bien ou mal ...? » Est-il bon ? Est-il méchant ?3 Voilà les questions qui intéressent Diderot. Ce sont, dit-il encore, des « questions qui appartiennent plus au caractère qu'à la logique ».

5 Si l'on prend au sérieux ce passage, Diderot aurait donc moins renoncé à la morale, que changé de stratégie. Tout en restant fidèle à ses intérêts d'homme vertueux, il porte l'attention sur un autre type de questions. La morale pour Diderot est, comme on l'avait noté, en premier lieu une affaire de caractères. Cela implique deux choses : que la morale est ancrée dans la vie pratique que nous connaissons, celle de tous les jours (la morale ne se fait donc pas dans les livres, pas même dans les livres de philosophie) ; et qu'elle est aussi plurielle que les caractères qui s'y rencontrent. Ainsi, le concept du caractère ne s'oppose pas seulement à la " logique » en tant que (pure) "théorie ». Il s'oppose également à la " loi » morale universelle pour rendre raison de la pluralité et de la diversité des caractères. Il n'a pas de parti pris pour la vertu, mais compte sur la méchanceté aussi bien que sur la bonté des hommes. En ceci, il est strictement « réaliste ». Mais le fait de remplacer, de façon programmatique, la « logique " par le " caractère " ne signifie pas pour autant que Diderot abandonnerait toute réflexion (philosophique). Au contraire, il ne renonce pas à la philosophie en tant que telle, mais change seulement de genre. Le caractère n'est pas, comme la logique, un procédé abstrait, il lui faut des situations et des histoires concrètes.

Le caractère demande donc une mise-en-narration. C'est pourquoi nous trouvons les réflexions de Diderot sur la morale non pas dans des traités (qu'il n'a pas faits), mais dans les contes. Les contes, ${ }^{4}$ et ce sera le sens du propos qui suivra, sont le lieu privilégié de la réflexion éthique de Diderot. Ils constituent une véritable étho-poétique, en ce qu'ils mettent en oeuvre une certaine expérience pratique du caractère, jointe à une réflexion théorique sur ses qualités - vices et vertus ! - éthiques et esthétiques. 


\section{Le champ sémantique du caractère}

7 Avant de commencer notre analyse des contes, essayons tout d'abord de rendre plausible une telle grille de lecture axée sur le caractère. Son évidence a dû être beaucoup plus grande pour Diderot qu'elle ne l'est actuellement pour nous : le concept (philosophique, anthropologique) du caractère vient sur lui de par deux traditions classiques, celle des moralistes du XVIIe siècle, en premier lieu, et celle de la philosophie morale antique dont héritent les premiers. Que Diderot soit un moraliste (pas comme les autres), ses commentateurs l'ont bien vu. ${ }^{5}$ Mais on peut pousser encore plus loin cette piste : au-delà de simples observations stylistiques concernant par exemple la forme de la maxime, on relève, par exemple, des parallèles frappants entre certains passages du Neveu de Rameau de Diderot et Les Caractères de La Bruyère, qui concernent l'objectif même de l'entreprise moraliste. Voyons par exemple le portrait du Neveu fait par MOI/Le philosophe au début de leur rencontre : MOI - Je n'estime pas ces originaux-là. [...] Ils m'arrêtent une fois l'an, quand je les
rencontre, parce que leur caractère tranche avec celui des autres, et qu'ils rompent
cette fastidieuse uniformité que notre éducation, nos conventions de société, nos
bienséances d'usage ont introduite. S'il en paraît un dans une compagnie ; c'est un
grain de levain qui fermente et qui restitue à chacun une portion de son
individualité naturelle. Il secoue, il agite, il fait approuver ou blâmer ; il fait sortir la
vérité ; il fait connaître les gens de bien ; il démasque les coquins ; c'est alors que
l'homme de bon sens écoute, et démêle son monde.

et comparons-là à la définition de l'approche moraliste chez La Bruyère :

Il s'en trouve un troisième ordre [d'auteurs en morale, K.B.], qui, persuadés que toute doctrine des moeurs doit tendre à les réformer, à discerner les bonnes d'avec les mauvaises, et à démêler dans les hommes ce qu'il y a de vain, de faible et de ridicule, d'avec ce qu'ils peuvent avoir de bon, de sain et de louable [...]. ${ }^{7}$

On note les ressemblances jusque dans l'emploi des termes (" démêler son monde ", " démêler dans les hommes ce qu'il y a de vain, de faible et de ridicule, d'avec ce qu'ils peuvent avoir de bon, de sain et de louable »). Diderot partage avec La Bruyère le même souci diagnostique : il s'agit de découvrir, de mettre à nu les caractères pour savoir à qui l'on a affaire dans la vie. ${ }^{8}$ Leur but est de mettre en lumière la vérité des caractères, de faire le tri, entre bons et mauvais, entre vices et vertus. Pour La Bruyère, l'entreprise s'arrête là : il vise, selon l'épistémologie classique, à établir une classification qui permet à son lecteur de s'orienter dans la société et qui n'est donc pas dépourvue d'éléments pédagogiques. ${ }^{9}$ Diderot, quant à lui, va un pas plus loin : pour lui, il ne s'agit pas seulement de classifier les caractères selon le schéma des types, mais de rompre " cette fastidieuse uniformité " imposée par la société moderne. Ainsi, non seulement son concept de caractère diffère fondamentalement de celui de La Bruyère en ce qu'il met l'accent sur l'originalité de l'individu ; mais aussi le style même de l'approche moraliste est un autre, plus dynamique, que chez son prédécesseur du XVIIe siècle. En fait, il ne s'agit pas seulement de comprendre les caractères, et donc d'une simple herméneutique, mais d'en provoquer l'émergence, de les produire. Le Neveu, ce " grain de levain qui fermente ", est un agent provocateur au sens strict du terme. Son rôle à lui (et c'est pourquoi le philosophe s'intéresse à ce personnage en dépit de son peu d' " estime ») est de découvrir des caractères (en tant qu'originaux) dans une société couverte du masque uniforme des bienséances. Avec Le Neveu de Rameau, Diderot franchit donc le pas d'une herméneutique vers une véritable poétique du caractère, centrée sur les vertus performantes du Neveu - 
le personnage du philosophe étant d'ailleurs le premier à éprouver l'étrange efficacité de son interlocuteur. ${ }^{10}$

Diderot s'inspire donc de La Bruyère, tout en prenant ses distances envers le moraliste classique. Celui-ci hérite à son tour d'une tradition ancienne, celle de la philosophie morale antique, représentée par Théophraste et son maître Aristote. Cette tradition, connue de Diderot, a dû avoir pour lui maints attraits. Tout comme les anciens, Diderot s'intéresse à la quête du bonheur et à la réalisation de la vie bonne (eudamonia). Il a donc dû être attiré par une philosophie centrée non pas, comme la philosophie morale moderne, sur la notion de « loi morale ", mais sur celle d'" éthos ", et donc d'emblée plus pragmatique et pluraliste que celle-là. ${ }^{11}$ Or, les différences sont aussi considérables : chez Aristote, par exemple, l'éthos désigne l'ensemble des dispositions acquises par habitude ; il ne s'agit donc pas (nécessairement) de quelque chose d'inné. ${ }^{12}$ Pour Diderot par contre, ce côté " génétique » (biologique) devient de plus en plus important (il suffit, pour s'en convaincre, de penser aux Éléments de physiologie et au Rêve de d'Alembert). Dans la Réfutation d'Helvétius, Diderot affirme sans ambivalence aucune que le caractère est un " effet de l'organisation ${ }^{13}$ ». Ainsi, tout en s'inscrivant dans la tradition éthique classique, Diderot donne-t-il une interprétation naturaliste au caractère. ${ }^{14}$ Par-là, il rejoint encore un certain courant de la philosophie antique, à savoir le matérialisme d'Héraclite, à qui nous devons la fameuse sentence que le " caractère est un destin ».

Voilà donc tous les éléments réunis pour un déterminisme sans faille en matière de caractère ? En vérité, les choses ne sont pas si simples. Tout d'abord, l'organisation de l'individu pour Diderot n'est pas fixe, mais flexible. Elle est, à un certain degré, ouverte à l'événement. ${ }^{15} \mathrm{En}$ plus, la tradition antique, à laquelle s'est formé l'esprit de Diderot, n'est en elle-même pas homogène. Elle nous a légué aussi le concept du caractère comme " persona ", c'est-à-dire comme " masque " ou " rôle ». Les " masques " que critique Diderot moraliste ne sont donc pas seulement opposés au caractère, mais ils font aussi partie d'un certain concept (théâtral) du caractère. De ce point de vue, le caractère est moins un destin irrévocable, qu'il est capable d'ironie. Entre le (véritable) caractère et son rôle, sa performance publique, existe toujours une faille, une non-coïncidence structurelle qui peut être interprétée comme une déficience, ou comme une liberté. Diderot, avec Le Paradoxe sur le comédien, a dédié un texte entier au caractère du comédien, l'acteur social par excellence. Le débat sur la liberté n'est donc pas fermé, mais ouvert. Nous allons y revenir dans nos analyses des contes.

12 Terminons ce parcours en jetant un rapide coup d'oeil dans l'Encyclopédie. La notion de caractère y joue un rôle important, et pas seulement pour des raisons de pure quantité (on compte au nombre de douze les entrées appartenant au " caractère ", plus quatre dans le Supplément de 1176). ${ }^{16}$ Écrits par au moins cinq auteurs distincts, ces articles couvrent une quantité de disciplines différentes, de la botanique jusqu'à la théologie, en passant par les techniques d'imprimerie et les métiers d'art (poésie, peinture). Au début, d'Alembert donne une définition générale du terme " caractère », soit :

Ce mot pris dans un sens général, signifie une marque ou une figure tracée sur du papier, sur du métal, sur de la pierre, ou sur toute autre matière, avec la plume, le burin, le ciseau ou autre instrument, afin de faire connaître ou désigner quelque chose.

13 Cette définition, qui s'en tient strictement à l'étymologie du terme (charassein en grec signifie marquer, tracer, imprimer), correspond par ailleurs assez exactement à ce qu'en disent les dictionnaires contemporains ${ }^{17}$ : le caractère au sens propre étant un signe, une 
empreinte qui sert à désigner quelqu'un (ou quelque chose) afin de le/la faire connaître. Notons que cette définition ne néglige ni les outils ni les supports - c'est-à-dire, tout le « matériau »- de cette empreinte (qu'il faut encore entendre dans un sens actif, comme processus de fabrication et résultat de ce processus ; je souligne en passant que c'est Diderot lui-même qui se charge des longues explications techniques dans l'article caracteres d'imprimerie). Par la suite, elle est variée selon la logique des différentes disciplines ; ainsi, l'on parle de " qualité essentielle » (C. en peinture), d'une " différence spécifique " (à propos du C. d'un ouvrage), d'une " manière propre " (C. en parlant d'un auteur) ; par rapport aux personnes, il est question d'une « disposition habituelle de l'âme " (C. en morale, $C$. des nations) et d'une " inclination ou passion dominante " (C. dans les personnages) ; pour en venir finalement aux significations plus nettement métaphoriques, tel l' " esprit de corps » et la " qualité invisible qui attire le respect ». C'est l'article Caractère - terme moderne de botanique qui reflète le plus clairement le désir de classification que nous avons déjà pu observer chez les moralistes classiques : «[...] le caractère d'une chose est ce qui la distingue essentiellement de toute autre chose ».

Peut-on, dans cet article vraiment pluri-disciplinaire sur le caractère, identifier des traits globaux ou communs, et qui nous aideraient à comprendre sa fonction dans les contes? Deux remarques semblent ici à l'ordre : premièrement, l'unité du caractère, si elle existe, ne peut que résider dans sa diversité même. Ainsi, tous les articles de l'Encyclopédie insistente sur le fait de la diversité des caractères ; celui-ci figurant d'ailleurs comme instrument heuristique servant à la connaissance (par distinction). Seconde remarque: le caractère, bien qu'il désigne en premier lieu l'essence des choses, renvoie tout aussi naturellement à leur mode de (re-)présentation. C'est une forme, une manière de dire ou de désigner ; il appartient donc, de par sa définition même, au monde des signes. Voici l'autre grand paradoxe du " caractère » : non seulement il permet de penser l'unité dans la diversité ; il est aussi situé au seuil de l'être du paraître, de la chose et de sa représentation. ${ }^{18}$ D'où s'explique aussitôt son attrait pour l'homme de lettres : au lieu d'y chercher (vainement) la cohérence d'une notion philosophique, Diderot tout au contraire tire profit de son hétérogénéité sémantique. Le caractère est pour lui un champ d'expérimentation ouvert ; il permet de réconcilier - sans les nier - des contradictions qui mèneraient à l'impasse au plan de la pensée logique. Comme signe entre signes, le caractère ne demande pas seulement la mise en forme littéraire ; en désignant à la fois l'essence intime des choses (ou des personnes) et leur représentation, il engage aussi une double réflexion, sur les êtres et sur les formes. C'est cette double réflexion, à la fois éthique et esthétique, qui, me semble-t-il, distingue les contes de Diderot dans le contexte narratif du XVIIIe siècle. Ceci dit, nous pouvons maintenant interroger à plus de loisir la " caractéristique » de ces contes. ${ }^{19}$

\section{Les contes comme école du jugement}

On commencera en relevant un aspect qui, jusqu'à présent, n'a pas encore reçu toute l'attention qu'il mérite, et qui concerne le rôle du jugement dans les contes. Les contes de Diderot ne mettent pas directement le lecteur en contact avec les personnages (les caractères), mais avec des jugements portant sur ces personnages et leurs actions. De ce point de vue-là, les contes de Diderot ne sont pas vraiment des contes (c'est ce qu'il dit d'ailleurs lui-même dans le titre de l'un d'entre eux), mais des méta-contes. Leurs véritables protagonistes sont le narrateur et son interlocuteur, qui s'entretiennent des 
personnes de leur connaissance en discutant le mérite (ou l'absence de mérite) de leurs actions. Tous les contes de Diderot sont pourvus d'un cadre narratif (soit en forme d'un dialogue qui ressemble à une conversation spontanée entre amis, soit en forme d'un échange de lettres comme dans Les deux amis de Bourbonne), qui confronte le lecteur avec une évaluation réflexive du sujet. ${ }^{20} \mathrm{En}$ insistant sur cette dimension (méta-)critique des contes, on pourrait dire aussi que ceux-ci mettent en scène leur propre réception (l'interlocuteur du narrateur figurant, sur le plan de la fiction, comme représentant du lecteur ${ }^{21}$ ). Par conséquent, les jugements dont il est question ici ne sont-ils pas exclusivement d'ordre moral : chez Diderot, la réflexion sur les caractères - l'éthique - est toujours liée à une réflexion esthétique. Citons en témoin Ceci n'est pas un conte, qui s'ouvre par une discussion sur ... l'art de faire des contes !22 Que la question « Est-il bon ? Est-il méchant? » ne s'applique pas seulement aux caractères, mais au texte en tant que tel, nous le comprenons quand le narrateur annonce, pour ce qui va suivre, un " mauvais » conte..$^{23}$ Bien sûr, on peut discuter du sens de cette phrase, comme d'ailleurs de celui du titre du texte - équivoque pour le moins. Mais pour l'instant, il suffit de noter que pour Diderot la réflexion critique (morale et esthétique) joue un rôle crucial dans les contes elle en constitue une partie intégrante.

Comment fonctionne, alors, la critique du jugement dans les contes ? Le schéma est presque toujours le même : comme dans Ceci n'est pas un conte, l'un des deux personnages du cadre (en général il s'agit de l'interlocuteur du narrateur), réagit aux histoires qu'on lui raconte de façon spontanée, émotive et non-réfléchie. Ainsi, en écoutant l'histoire du malheureux Tanié, trahi par la belle et cruelle Madame Reymer, exècre-t-il spontanément les femmes. ${ }^{24}$ Même chose, quand il apprend ce qui s'est passé entre Gardeil et Mademoiselle de La Chaux. Gardeil est un scientifique égoïste qui exploite sa compagne et l'abandonne quand, exténuée par le travail, elle a perdu ses attraits. Cette fois-ci, pourtant, l'interlocuteur prend parti pour Mademoiselle de La Chaux (et donc pour les femmes). À chaque fois, il semble suivre naturellement la "morale " dominante de chaque histoire - même si l'une illustre la méchanceté des femmes et l'autre celle des hommes. Au narrateur revient donc le rôle de lui faire comprendre que les choses sont en vérité plus complexes. Ainsi, lorsque l'interlocuteur s'exclame, à propos de Gardeil : « Oh les hommes! les hommes! », il lui répond : «Vous regardez le méchant et vous ne voyez pas tout à côté l'homme de bien ${ }^{25}$ ». Et effectivement, dans le second conte figure aussi un homme de bon coeur, le docteur Camus, qui s'occupe de Mademoiselle de La Chaux quand elle est seule et malade. Ainsi, Ceci n'est pas un conte offre un bel exemple de la technique narrative de Diderot : le narrateur presente non pas un conte, mais deux, à tendance morale contraire ; et il soumet son interlocuteur à une véritable école de jugement en lui signalant que, quant au caractère (des hommes et des femmes), les choses ne sont pas si simples qu'il ne l'avait d'abord pensé.

17 La même technique (et peut-être les mêmes interlocuteurs ?) se retrouve aussi dans Madame de La Carlière. Ce conte, qui forme la deuxième pièce d'une série de trois, traite lui aussi d'un couple malheureux et, plus concrètement, du thème de l'infidélité sexuelle. Encore une fois, en tant que lecteurs, nous n'avons pas affaire directement aux personnages, mais aux jugements qu'on a passé sur eux « dans le monde ». Cette fois-ci, pourtant, ces jugements proviennent d'une instance qui n'existait pas dans Ceci n'est pas un conte, mais qui prend un essor inouï dans la société du XVIIIe siècle : l'opinion publique. L'interlocuteur, faisant toujours le rôle du naïf, s'avère un adepte peu critique de l'opinion publique quand il juge sévèrement le mari de Madame de La Carlière, le 
chevalier Desroches, en le traitant de «fou qui a subi toutes sortes de métamorphoses ${ }^{26}$ ». Le narrateur, quant à lui, ne cache pas son dédain de l'opinion publique, qu'il appelle « cette foule imbécile ${ }^{27} »$. Loin d'être - comme le voudrait la propagande des Lumières - un tribunal objectif de la raison, l'opinion publique est pour lui irrationnelle et profondément partiale, une instance d'autant plus redoutable que son verdict est chargé de conséquences pour celui qui en devient la cible. Il s'applique donc à motiver, aux yeux de son interlocuteur, les différentes «métamorphoses » (professionnelles et amoureuses) de Desroches, en lui expliquant les raisons de celui-ci que ne connaît pas le public et qui sont, en général, beaucoup plus intègres qu'on ne l'aurait pensé au premier abord.

La stratégie du narrateur consiste donc à rectifier le jugement (qui est en vérité un préjugé) de son interlocuteur en lui racontant des « faits » que celui-ci ne connaissait pas. Voilà à quoi sert, dans ce contexte, l'histoire de Desroches : elle nous donne une idée de son caractère moral indépendante de son image, de son caractère public. ${ }^{28}$ En même temps, la position du narrateur est ici beaucoup moins équivoque que dans le premier conte : non seulement il ne cache pas son mépris du jugement public, mais il n'hésite pas non plus à réprimander sévèrement son interlocuteur quand celui-ci essaie de se trouver des excuses. ${ }^{29}$ Grâce à cette double intervention du narrateur, l'interlocuteur se voit finalement obligé de réviser sa position : il apprend non seulement à se méfier du bruit public, du " qu'en-dit-on ", mais aussi, et ce sera la tendance de la deuxième partie du conte, à reconnaître - à prédire même ! - les lois (de formation, de développement) de l'opinion publique qui, de façon paradoxale, s'avère être au moins aussi « inconséquente » que les hommes qu'elle condamne. ${ }^{30}$

19 Ayant passé en revue la technique narrative de Diderot, nous sommes maintenant à même de comprendre pourquoi Diderot s'en prend aux jugements qui font le sujet de ses contes. Il les critique en tant que représentations (sociales, publiques, en tout cas néfastes) auxquelles s'opposerait le véritable caractère des personnes. Les jugements, comme d'ailleurs les lois morales sur lesquelles ils reposent et auxquelles ils ressemblent de par leur forme apodictique, font partie de tout l'artifice culturel que semble redouter le narrateur des Trois Contes. ${ }^{31}$ Ce sont, si nous nous en tenons à l'antique opposition de physis vs. nomos, des "fictions ", c'est-à-dire, des fabrications humaines qui, tout compte fait, n'ont pas grand-chose à voir avec la « réalité ». Non seulement les jugements que nous portons les uns sur les autres ne sont-ils pas en accord avec le caractère des personnes ; dans le pire des cas, ils le falsifient et le corrompent. ${ }^{32}$ C'est exactement ce que montre la fameuse « carrière » du chevalier Desroches dans l'opinion publique : non seulement son image publique n'a rien à voir avec son véritable naturel, mais cette image est aussi contradictoire et gouvernée par des intérêts étrangers au caractère en tant que tel. ${ }^{33}$ La critique que présente Diderot dans les contes sert donc surtout à restreindre, autant que possible, l'influence néfaste que peuvent exercer les jugements moraux dans la vie des hommes : «l'inconséquence » dont il est question dans le sous-titre de Madame de La Carlière est à entendre dans un sens normatif (de jure), non pas comme un simple constat (de facto).

Est-ce qu'il faut, pour autant, renoncer à tout jugement moral ? C'est effectivement ce qu'on pourrait croire en lisant la fin de Ceci n'est pas un conte. Quand les choses entre les deux interlocuteurs sont devenues compliquées au point de rendre toute entente impossible, le narrateur propose la réflexion suivante :

Mais on me dira peut-être que c'est aller trop vite que de prononcer définitivement sur le caractère d'un homme d'après une seule action ; qu'une règle aussi sévère 
réduirait le nombre des gens de bien au point d'en laisser moins sur la terre que l'évangile du chrétien n'admet d'élus dans le ciel ; qu'on peut être inconstant en amour, se piquer même de peu de religion envers les femmes sans être dépourvu d'honneur et de probité ; qu'on n'est le maître ni d'arrêter une passion qui s'allume, ni d'en prolonger une qui s'éteint ; qu'il y a déjà assez d'hommes dans les maisons et les rues qui méritent à juste titre le nom de coquins, sans inventer des crimes imaginaires qui les multiplieraient à l'infini. On me demandera si je n'ai jamais ni trahi, ni trompé, ni délaissé aucune femme sans sujet. Si je voulais répondre à ces questions, ma réponse ne demeurerait pas sans réplique, et ce serait une dispute à ne finir qu'au jugement dernier. ${ }^{34}$

21 Après avoir longuement discuté, avec son interlocuteur, le cas des deux couples, le narrateur procède ici, comme par hypothèse, à une mise en question générale du jugement. La tendance de son propos est assez claire : c'est le jugement qui fait les criminels, et la réalité (lisons : la réalité physique, la sexualité des hommes) n'est pas faite pour être soumise à des lois morales. Mais le texte ne s'arrête pas là. Tout d'un coup, le narrateur interpele directement son lecteur : « Mais mettez la main sur la conscience, et dites-moi, vous, monsieur l'apologiste des trompeurs et des infidèles, si vous prendriez le docteur Toulouse pour votre ami ?»

C'est donc au lecteur, que le narrateur s'adresse ici comme « Monsieur l'apologiste des trompeurs et des infidèles " en raison de son scepticisme (présumé) en matière de morale, que revient l'honneur - ou disons plutôt : la tâche - de prononcer un jugement final..$^{35}$ Il n'y a donc pas, chez Diderot, en dépit de toutes ses réserves, de " suspension of judgment » à la manière des post-modernes. Mais ce jugement, non seulement il incombe au lecteur de le formuler (le texte laisse un blanc plus ou moins suggestif...) ; il est clair aussi qu'il ne peut, en aucun cas, prétendre à l'objectivité comme le faisaient les autres verdicts critiqués dans le conte. La question adressée au lecteur n'est pas « Est-il est bon ? Est-il est méchant ? ", mais bien « Est-ce que vous [le] prendriez pour ami ». Poser la question de cette façon implique non seulement que la réponse sera nécessairement subjective (« l'amitié » renvoyant au domaine des préférences subjectives, non pas à celui des normes exactes) ou qu'elle aura pour unique fonction de donner une orientation pratique. Savoir si l'on peut vivre avec quelqu'un, en l'ayant pour ami, est une affaire personnelle qui se décide au niveau de la vie pratique et qui est, pour cette raison même, dépourvue de toute portée normative. ${ }^{36}$ Mais le point essentiel ici est encore un autre : c'est le fait que la question discutée tout au long du conte par rapport aux caractères (des personnages) ne saurait être résolue, en définitive, que par le caractère (du lecteur) : en donnant une réponse à la question du narrateur, celui-ci va révéler ses préférences, ses sympathies (ou absences de sympathies), bref, son caractère. Ainsi, en guise de clôture, c'est le caractere du lecteur qui se trouve impliqué dans le débat : à lui la décision.

\section{Une morale « fondée » sur la nature?}

Essayons un premier petit bilan. Comme nous l'avons vu dans la section précédente, Diderot, dans ses contes, met en oeuvre une critique du jugement moral qui est aussi (et en même temps) une critique de la fiction. Plus exactement, Diderot critique les jugements en tant que fictions, c'est-à-dire comme faisant partie de l'artifice culturel qu'ont institué les sociétés civilisées, et qui donneraient lieu à des " crimes imaginaires », à une « dépravation artificielle » qui, en tant que telle, n'existerait pas à l'état de nature. Nous nous sommes encore référés à un élément de la philosophie antique, la fameuse 
opposition physis vs. nomos pour indiquer quelle est, chez Diderot, la fonction de la notion de caractère : tandis que les jugements (les normes, lois ...), étant des positions plus ou moins arbitraires, représentent le domaine des nomoi, le caractère quant à lui appartient au domaine de la physis; il désigne le «naturel » de l'homme - soit le côté de son être moral qui échappe à la contingence. Sur le caractère, il parait donc qu'on pourrait fonder une morale non-arbitraire, une " morale physique » qui s'ordonnerait non pas sur ce qu'imaginent les hommes, mais sur ce qui est vraiment (dans la nature). Voilà, semble-til, le projet qui fascine Diderot, et qu'il met à l'épreuve dans ses contes.

Que ce soit un projet extrêmement difficile, Diderot ne prend aucun soin de le cacher. Bien au contraire : son esprit, qui se plaît à peser le pour et le contre, trouve presque toujours dans les difficultés des ressources nouvelles. Les deux contes discutés ci-dessus ( Ceci n'est pas un conte, Madame de La Carlière) ont révélé des tensions inhérentes au caractère : face à la simplicité apparente des jugements normatifs, ils mettent en relief, premièrement, la complexité des caractères (surtout, mais pas exclusivement, pour les caracteres génériques, p.ex. " les hommes ", " les femmes ») et, deuxièmement, leur historicité (un problème discuté surtout par rapport au motif de la constance en amour). Non seulement les caractères sont, de par leur nature même, intrinsèquement complexes et hétérogènes, ils ont aussi tendance à changer avec le temps. ${ }^{37}$ À quel point cela rend difficiles les relations sociales, les deux contes le montrent en toute franchise. À ces deux premières observations sur le caractère s'ajoutent d'autres, plus sérieuses, avec le Supplément au Voyage de Bougainville. Ce conte, qui semble au prime abord faire l'apologie d'une vie insouciante et libre selon l'état de nature, propose, à y regarder de plus près, une réflexion philosophique ambitieuse sur les difficultés liées au projet de fonder une morale sur la nature. L'utopie, dans ce texte, a des fissures graves ; l'empire même de la nature n'est pas sans équivoques : car, non seulement la loi de la nature, appliquée strictement, fait preuve d'une certaine tendance despotique,$^{38}$ en révélant par là sa complicité secrète avec les institutions de la civilisation européenne ; mais il semble au moins tout aussi difficile de dériver, de la nature en tant que telle, des directives concrètes pour la vie en société. En témoigne la discussion entre les deux interlocuteurs à la fin du texte, quand ils essayent de soumettre les pratiques galantes de leur société au " test » de la nature..$^{39}$ À la fin, quand tout semble dit, ces deux personnages décident non pas de se rendre sur le champ à Otaïti, mais de se conformer volontairement et provisoirement aux conventions de leur société, tout en essayant d'en réformer les abus selon les indications de la nature.

Les ambivalences structurelles de ce texte (il s'agit d'une critique de la fiction par le moyen de la fiction !) et la décision finale des deux interlocuteurs montrent qu'on ne saurait réduire trop facilement la position de Diderot à un naturalisme sans faille. Tout n'est pas, et ne peut pas être, nature. Bien qu'il reconnaisse, dans certaines limites, l'autorité normative de la nature, la position de Diderot reste culturaliste au fond. Le fait que les deux protagonistes du Supplément prennent une décision délibérée en faveur de la civilisation, montre bien qu'il n'y a, de leur part, aucun fatalisme vis-à-vis l'empire de la nature. Cette position, qui est aussi celle de Diderot, devient plus cohérente encore quand on la met en rapport avec sa conception du caractère. D'après tout ce que nous en savons, le caractere non plus ne saurait être réduit à la simple physique. Ce n'est pas une simple donnée, une tendance naturelle et matérielle, mais une organisation complexe qui comporte toujours des éléments culturels. Ainsi, au caractère, en tant que « naturel » de l'homme, il convient de donner une forme ; il peut et doit être l'objet d'une formation. 
N'oublions d'ailleurs pas que, chez les anciens, le caractère était au centre d'une véritable esthétique de l'existence. ${ }^{40} \mathrm{Chez}$ Diderot, on trouve encore les vestiges d'une telle approche : pensons p. ex. à l'actrice décrite dans le Paradoxe sur le Comédien et qui cherche, lors de ses minutieuses répétitions, à se conformer à un modèle idéal. ${ }^{41}$ Or, le concept même de s'exercer (mentalement, physiquement) pour se conformer à un modèle quelconque montre clairement que le caractère pour Diderot n'est pas un simple fait, mais une forme qu'on peut se donner, un rôle qu'il faut remplir (de vie).

Toujours est-il que les réflexions de Diderot sur l'esthétique de l'existence sont, en général, liées à une autre figure, bien connue, celle du génie (noir), comme le père Hudson, Madame de La Pommeraye etc. Entre l'actrice du Paradoxe et Madame de La Pommeraye, il y a d'ailleurs maints parallèles. Madame de La Pommeraye joue, elle aussi, un rôle, elle se donne une forme extérieure modelée sur une idée qu'elle s'est faite : celle de sa vengeance sur son mari, le Marquis des Arcis, qui a cessé de l'aimer (sans pourtant la trahir). Quant au sort qui est le sien, Madame de La Pommeraye est comme une soeur jumelle de Madame de La Carlière : mais là où celle-ci préfère souffrir en silence, La Pommeraye relève le gant et opte pour le combat. À un destin hostile, elle oppose sa propre détermination, sa volonté féroce, qui est d'abord une volonté de nuire le plus que possible à cet homme à qui elle a confié sa tranquillité et sa réputation, et qui l'abandonne au moment précis où elle n'a plus rien à perdre. Ainsi, chez Madame de La Pommeraye, le caractère n'est pas seulement une forme, mais une véritable force ${ }^{42}$ : son " génie ", qu'admire - entre autres - le narrateur du roman, ${ }^{43}$ ne consiste pas dans la seule ingéniosité avec laquelle elle manipule les gens de son entourage, mais dans sa volonté de triompher, dans sa détermination presque folle de résister au " sort » (incarné par les sentiments ou plutôt le défaut de sentiments de la part du mari). ${ }^{44}$ Madame de La Pommeraye est une femme qui ne veut surtout pas être victime (du sort) ; c'est pourquoi, ayant perdu son amour, elle fait tout pour rester quand même la maîtresse des sentiments de son mari, en les transférant sur un objet qu'elle considère indigne de son affection.

Concluons, pour le moment, que dans l'histoire de Madame de La Pommeraye, rapportée par l'hôtesse d'auberge en plein milieu de Jacques le Fataliste et son maître, Diderot met en scène l'antique combat du caractère et du destin. ${ }^{45}$ C'est pourquoi ce " conte ", qui n'en est pas un, comme tous les contes de Diderot, doit nous intéresser ici : il constitue le lien entre les contes, avec leurs variations sur le caractère, et le sujet du roman, le fatalisme. Ici se manifeste aussi, avec tout son éclat, la virtuosité dialectique de la pensée de Diderot : entre le caractère et le destin, il y a, comme le montre déjà la sentence d'Héraclite ( «le caractère est un destin »), une filiation secrète. Ce sont, tous les deux, des forces determinantes de la vie humaine : détermination extérieure, dans le cas du destin, détermination intérieure, dans celui du caractère. Ainsi, chez Diderot, on ne sort pas facilement du règne de la détermination, plus ou moins total; mais ce que ce texte nous donne quando même à penser, c'est la lutte des déterminations, internes et externes, quand l'homme (ou la femme) de caractère s'avère un adversaire digne du destin. ${ }^{46} \mathrm{Si}$ on ne peut donc pas, à propos du caractère, parler de « liberté " proprement dite, on peut, tout au moins, y voir un autre type de détermination, par la volonté, ${ }^{47}$ et qui s'oppose aux déterminations extérieures auxquelles sont soumis les hommes.

Quant au succès de cette fabuleuse "révolte " contre le destin, il est marqué, dans le roman, par une étrange indétermination : lorsque le plan diabolique de Madame de La Pommeraye a été mis en oeuvre et qu'elle touche au moment de son triomphe, il arrive 
quelque chose de tout à fait imprévu : quand le marquis des Arcis apprend qui est en vérité sa nouvelle femme (une ancienne prostituée), il ne la renvoie pas, mais lui propose de passer leur vie ensemble et de vivre en couple heureux. À cette femme échoit donc le bonheur qui a été refusé à Madame de La Pommeraye. Cela a de quoi surprendre. Cependant, les raisons qui motivent le comportement du Marquis restent cachées aux lecteurs. ${ }^{48}$ Est-ce qu'il a, en optant pour la fidélité envers sa nouvelle femme, tout simplement subi un autre tour d'esprit, c'est-à-dire, est-ce qu'il est encore le jouet de ses passions, ou est-ce qu'il a, au contraire, décidé, de façon délibérée, de se comporter en homme d'honneur envers elle ? Le silence de Diderot là-dessus est significatif, et donne libre cours à la spéculation : dans le premier cas, nous aurions affaire à une ironie du sort ; dans le second cas, à une décision éthique.

Le projet de vengeance de Madame de La Pommeraye échoue donc, mais les raisons de cet échec restent dans le noir. Cette indétermination au niveau de l'interprétation du texte fait partie de l'art narratif de Diderot : elle y introduit un élément qui n'est étranger ni au destin, ni au caractère, et qui joue un rôle absolument central dans les contes : l'ironie. L'ironie affecte la morale de ces contes de fond en comble. Quand par exemple l'interlocuteur dans Ceci n'est pas un conte commente l'indifférence des prêtres, qui refusent de secourir Mademoiselle de La Chaux dans sa détresse, en disant «Cela est dans la règle ", cette réponse est bien sûr ironique. ${ }^{49}$ Elle est ironique en tant qu'elle présente un semblante de morale, lorsqu'en fait il s'agit tout simplement de constater un comportement immoral, devenu une normalité dans la société. Ironie il y a également dans le cas de Madame de $\mathrm{P}^{* * *}$ dans Les deux amis de Bourbonne, quand cette dame, qui était d'abord pleine d'admiration et de compassion pour les deux amis, remercie son directeur de conscience, le curé Papin, de l'avoir " éclairée " sur le véritable sens de la charité chrétienne..$^{50}$ Mais l'ironie chez Diderot opère aussi dans le rapport du texte avec son lecteur : il est essentiel, pour l'effet éthique de ces contes, que le lecteur ne soit pas déterminé entièrement par les avis des personnages fictifs. ${ }^{51}$ Dans le cas de Madame de $\mathrm{P}^{* * *}$, par exemple, il est (sous-)entendu que nous ne suivions pas son jugement, mais que nous prenions nos distances envers elle, tout comme elle s'est auparavant distanciée des deux amis. Le lecteur fictif (represente dans ce cas par Madame de P***) n'est pas identique avec le lecteur implicite ${ }^{52}$ - la position de ce dernier étant laissée délibérément en suspens. C'est pourquoi la «morale » de ces contes s'écrit souvent dans le silence, dans les non-dits du texte.

30 Chez Diderot donc, l'ironie fait pendant à la force ; elle repose sur la non-identité de l'être et du paraitre, et en tire toute son efficacité. L'ironie est aussi, comme on l'avait déjà suggéré, une qualité inhérente au caractère. Entre le (véritable) caractère et le masque, entre le naturel et le rôle (public, social, théâtral), il y a non-identité structurelle. Cette non-identité peut être un scandale - et une ressource. Chez Diderot, c'est parfois les deux à la fois..$^{53}$ Dans la vie pratique, il paraît qu'il s'est souvent scandalisé des gens faux, en particulier des simulacres de la vertu. Par contre, dans l'histoire de Madame de La Pommeraye et autres contes, il exploite l'effet dramatique qui résulte des déterminations opposées, et met à profit l'indétermination particulière de l'ironie pour offrir à son lecteur la possibilite d'un positionnement éthique. À ce tableau, L'Entretien d'un père avec sens enfants, texte qui traite - comme le Neveu de Rameau - des "idiotismes moraux ", ajoute encore un nouvel aspect. À la question comment il faut juger le cas problématique d'un calzolaio qui, désespérant de l'ordre public représenté par le Vice-roi, prend en main lui-même l'exécution des criminels, le personnage MOI/Diderot fils donne la réponse 
suivante: " Je condamnerai le Vice-roi à prendre la place du savetier et le savetier à prendre la place du Vice-roi ${ }^{54}$ ». Ici, le rapport entre le caractère de la personne et sa fonction publique est appelé à résoudre jusqu'au problème d'une société juste. Au lieu de juger, voire de condamner les caracteres sur fond de normes abstraites, mieux vaut les mettre en accord avec eux-mêmes, en leur permettant d'exercer la fonction (la capacité) qui est en harmonie avec leur dons naturels (leurs capacités). De cette harmonie, Diderot est convaincu, résultera non seulement le bonheur des individus, mais aussi celui de la société. ${ }^{55}$

Dans L'Entretien, nous voyons donc Diderot soucieux d'élaborer une dialectique plus complexe encore que celle, relativement simpliste, de la nature et de la culture, qui faisait l'objet du Supplément au Voyage de Bougainville. C'est une dialectique vraiment pluraliste, basée sur les "idiotismes moraux ", c'est-à-dire les différences spécifiques des individus et des conditions, qui sont aussi nombreux que les caractères mêmes. La diversité des caractères, qui restera toujours incompréhensible du point de vue de la loi (universelle, unique) de la morale, parce qu'on n'y saurait voir que des déviances illégitimes (des " idioties » au sens étymologique du terme), devient ici une véritable ressource de la pensée éthique. L'Entretien d'un père avec ses enfants représente, en conséquence, un état très avancé de la pensée morale de Diderot. Non seulement ce texte montre-t-il que les personnages jugent les uns des autres en fonction de leur " condition " particulière ; il nous permet aussi d'apprécier cette diversité comme une véritable valeur. L'essentiel, du point de vue social, est que les diverses fonctions soient bien orchestrée entre elles, et qu'il y ait harmonie entre le naturel des individus et la fonction publique exercée par eux. Ceci nous suggère une dernière observation, valable pour tous les textes analysés jusqu'ici : que "l'harmonie " (harmonie avec soi-même, avec les autres) est une notion-clé de la pensée morale de Diderot, comme le montre la fonction véritablement décisive qu'il lui attribue dans les débats éthiques - qu'il s'agisse des personnes (harmonie entre les caractères), des institutions (harmonie entre la nature et la culture) ou de l'organisation sociale (harmonie entre le caractère et la fonction publique). L'harmonie, et c'est là encore une idée ancienne (platonicienne, pour être précis, transmise à Diderot par l'oeuvre de Shaftesbury), est la réponse que permet de donner l'esthétique de l'existence à la problématique de la morale.

\section{Enjeux littéraires}

Evidemment, la réflexion sur le rapport entre " caractères » et « conditions ", telle qu'elle figure dans L'Entretien d'un père avec ses enfants, n'est pas nouvelle chez Diderot. Nouvelle est plutôt la configuration harmonique entre les deux, qui procède à son tour d'une nouvelle appréciation, plus positive, du caractère en tant que tel. On sait que, dans les années 50 , à l'époque du drame bourgeois, Diderot opposait " caractère » et " condition ", en donnant la préférence à la dernière.$^{56}$ Le contexte de ses réflexions à l'époque était celui de l'intérêt du spectateur ; le souci principal de Diderot, en tant qu'auteur dramatique, celui d'assurer la sympathie des spectateurs envers les personnages et leurs problèmes respectifs. Il donnait alors la préférence à la condition sur le caractère, parce qu'il croyait y déceler un potentiel d'identification plus grand : " Pour peu que le caractère fût chargé, un spectateur pouvait se dire à lui-même, ce n'est pas moi. Mais il ne peut se cacher que l'état qu'on joue devant lui ne soit le sien ; il ne peut méconnaître ses devoirs. Il faut absolument qu'il s'applique ce qu'il entend ${ }^{57}$ ». L'argumentation de 
Diderot, dans ce passage de L'Entretien sur le Fils Naturel, obéit évidemment à une logique universaliste : tandis que le caractère est, par nature, trop singulier pour donner lieu à une véritable identification de la part du spectateur, la condition représente, quant à elle, un « universel singulier » qui permet l'identification d'un nombre plus important de spectateurs. ${ }^{58}$ C'est donc en sa qualité de pédagogue que Diderot parle à cette époque : tandis que vices et vertus sont des affaires particulières (en, en conséquence, l'affaire des particuliers), le spectateur, étant forcé « d'appliquer à lui ce qu'il entend », ne pourrait " méconnaître » ceux de ses « devoirs » qui sont liés à la condition.

Lorsqu'il écrit ses contes, vers la fin des années 70, Diderot aura abandonné le ton de prédicateur ; à part cela, les préoccupations de l'écrivain sont restés plus ou moins les mêmes. C'est ce que montre de façon exemplaire la fin du conte Les deux amis de Bourbonne , où Diderot fait, dans une espèce de post-scriptum, le portrait du conteur historique : « Celui-ci se propose de vous tromper, il est assis au coin de votre âtre ; il a pour objet la vérité rigoureuse ; il veut être cru : il veut intéresser, toucher, entraîner, émouvoir, faire frissonner la peau et couler les larmes ; effets qu'on n'obtient point sans éloquence et sans poésie ${ }^{59}$ ». Même pour le « conteur historique » qu'il est devenu entre-temps, il s'agit donc toujours d'éveiller l'intérêt du lecteur. Bien qu'il ne prêche plus aux autres leurs devoirs, mais se contente de les inciter à une réflexion critique sur la morale en tant que telle, Diderot est bien conscient qu'il lui faut toujours intéresser ses lecteurs ; c'est une " condition "vraiment essentielle pour tout ce qu'il entreprend comme historienthéoricien de la morale. Et puisque, comme on l'avait noté au début, les contes font toujours aussi un peu leur propre critique, il n'est pas surprenant que l'on retrouve, dans le sujet de ce conte, une réflexion explicite sur les conditions de la participation affective. Ainsi, lorsque le curé Papin demande sournoisement à Madame de $\mathrm{P}^{* * *}$ : « J'ignore [...] quel intérêt vous pouvez prendre à deux brigands, dont tous les pas dans ce monde ont été trempés de sang ${ }^{60} "$, nous comprenons aussitôt qu'il en va toujours du thème de l'intérêt, et que celui-ci reste liée, chez Diderot, à la condition (identifiée ici, pour la première fois, à une problématique sociale). Ce qui a pourtant changé depuis le temps du drame bourgeois, c'est l'appréciation désormais positive de deux « brigands », qui, en tant que caractères mixtes, ne nous intéressent pas tant à cause de leurs vertus (qui sont pourtant incontestables), mais à cause de leurs défauts non moins évidents et qui en font partie intégrante.

Dans Les deux amis de Bourbonne, l'intérêt du lecteur se porte donc sur ce qu'on pourrait appeler, avec une certaine justesse étymologique, deux « idiots » (deux caractères hors la norme). Mais les lecteurs, dans cette histoire, ne sont pas les seuls intéressés : grâce à l'échange de lettres qui constitue le cadre narratif de ce conte, nous avons affaire à plusieurs narrateurs concurrents et impliqués, chacun à sa manière, dans le jeu des évaluations caractérielles. Bien sûr, l'exemple le plus frappant est celui, déjà cité, du curé : sa lettre sert, plus ou moins ouvertement, à discréditer les deux amis, afin de s'emparer de la somme que voulait leur offrir Madame de $\mathrm{P}^{* * *}$. Mais ce qui est vrai pour le curé l'est aussi pour tous les autres narrateurs : chacun veut obtenir, de la part de son lecteur, un crédit quelconque ; c'est-à-dire il veut amener son lecteur à investir ses ressources (affectives, financières, en tout cas : matérielles) dans son histoire. De cette vérité générale, Les deux amis de Bourbonne nous livre une illustration tout à fait plastique en présentant, au moyen des lettres, plusieurs narrateurs différents et leurs portraits toujours partiaux - des deux protagonistes. Ce qu'ils ont tous en commun, pourtant, c'est que personne n'y raconte pour rien : même la veuve d'olivier, dans sa misère, n'est pas un 
narrateur désintéressé. Voilà au moins ce que nous pouvons inférer de la réaction - en toute apparence complaisante - de Madame de $\mathrm{P}^{* * *}$ à son récit : « Nous avons parlé d'elle, et j'espère que notre recommandation ne lui aura pas été inutile ${ }^{61}$ ». L'intérêt que mobilise le narrateur en éveillant la sympathie de son lecteur se paie donc pour lui en argent comptant, et c'est pourquoi tous les narrateurs, dans ce conte, sont des narrateurs intéressés.

La réflexion méta-narrative qu'on peut discerner dans Les deux amis de Bourbonne mène donc infailliblement à une mise en cause du caractère du narrateur. Ainsi, chez Diderot, non seulement le caractère du lecteur, mais aussi celui du narrateur se trouve implique dans l'histoire. Tel est, par ailleurs, le sens des nombreuses apparitions de "Diderot " dans ses propres contes. Loin d'y voir un simple souci d'authentification de la part de l'auteur, et qui ferait partie du (proto-)« réalisme » de Diderot, cette technique renvoie plutôt à l'agenda etho-poétique des contes. Comme le dit de manière un peu oblique la poétique de Les deux amis de Bourbonne, Diderot s'y démarque d'une esthétique intégriste, qui opposerait nettement (comme le fait par exemple l'esthétique classique) le monde réel et le monde idéal de l'art. ${ }^{62}$ Par conséquent, et le lecteur et le narrateur sont ce que William Edmiston a appelé, sans peut-être en discerner toute la signification, des " participating personage(s) » (en tant qu'ils ne sont pas étrangers au sujet du conte, mais en font partie intégrante). ${ }^{63}$ Leur implication dans l'histoire correspond toujours, d'un point de vue moral, à une corruption morale : le caractère du narrateur n'étant nullement plus « intègre » que celui de son personnel fictif. C'est d'ailleurs exactement ce qu'avait suggéré - en toute ingénuité - le narrateur de Ceci n'est pas un conte, quand il nous avait signalé que lui-aussi, il n'avait pas toujours été un modèle de fidélité envers les femmes. ${ }^{64}$ La réflexion sur le caractère du narrateur est poussée au comble dans Mystification, premier conte de Diderot après L'oiseau blanc, conte bleu et dont la portée programmatique égale celle de Ceci n'est pas un conte. Dans Mystification, Diderot ne figure pas seulement comme personnage ; il y joue, face à Mademoiselle Dornet, son propre rôle, voire celui de " philosophe » et de " tête forte ${ }^{65}$ ». D'une certaine manière, Diderot et son complice Desbrosses sont des conspirateurs non moins " intrigants " que Madame de La Pommeraye ; mais il y aussi a, chez ces deux personnages, une ironie manifeste qui fait virer l'histoire dans le genre comique. ${ }^{66}$ Desbrosses, dans ce contexte, représente un comique assez traditionnel, lorsque, par exemple, en faisant le diagnostic du caractere de Mademoiselle Dornet, il s'en prend à la " physionomie » de cette demoiselle un peu trop directement ; au personnage de Diderot par contre revient une ironie plus fine : dans ses interactions avec la Dornet, qu'il essaye de convaincre de l'influence néfaste de deux portraits, il ressemble beaucoup au conteur historique qui, lui aussi, est « en même temps [...] véridique et menteur ${ }^{67}$ ». Ceci dit, nous pouvons tenter l'hypothèse que, dans ce conte, Diderot, grâce au personnage du même nom, met en scène sa propre conduite en tant que conteur : le narrateur s'implique lui-même dans l'histoire, en y jouant son propre rôle (celui de « Diderot intrigant »). D'un côté, cela lui permet de mettre en cause sa conduite, d'en faire le sujet d'une interrogation éthique un peu à la manière de Est-il bon? Est-il méchant ? D'un autre côté, et grâce à l'ironie qui est la sienne, cela lui permet de faire aussi exactement le contraire : au niveau tout à fait pratique qui est celui des interactions décrites dans le conte et effectuées par lui, Diderot se joue de ses interlocuteurs, que ce soient Mademoiselle Dornet (sur le plan de l'histoire) ou le lecteur (sur le plan du discours). 
Mystification réunit donc, au sein d'un seul conte, tous les équivoques de l'art narratif de Diderot. C'est à la fois une réflexion éthique sur le caractère (a-)moral du narrateur, et un jeu esthétique libre, dégagé de toute réflexion morale. Dans ce texte où tout tourne autour les qualités (présumées) vicieuses de quelques "simulacres ", la question posée par Mademoiselle Dornet par rapport aux manoeuvres de Desbrosses : « quelle confiance [peut-on] avoir dans un art qui m'a toujours paru suspect ${ }^{68}$ » fait directement écho à celle, déjà citée, du curé Papin : « Quel intérêt pouvez-vous prendre à deux brigands... ». En tant que lecteurs, cette question ne saurait nous laisser indifférents, puisque nous savons que dans ce texte, où elle prend le rôle de l'interlocutrice de Diderot, Mademoiselle Dornet représente aussi notre propre image. Effectivement, quelle confiance pouvons-nous faire à un conteur tel Diderot qui, tout en nous posant des questions éthiques sérieuse sur l'art et par l'art, déjoue de ces mêmes questions par une désinvolture ironique sans pareil ? Qui essaie de nous intéresser à ses histoires tout en nous montrant ouvertement qu'il est lui-même un narrateur intéressé et peu intègre ? Quelle confiance peut-on faire à un tel caractère? Au caractère en tant que tel ?

La réponse est pourtant simple : aucune, si nous le prenons pour un « fondement » solide, à la manière des objets physiques. Avec le caractère, nous sommes toujours déjà dans le domaine de la morale, qui est aussi, comme nous le savons, celui de l'art. Par conséquent, la pensée morale de Diderot, telle que nous l'avons lue dans les contes, est prise dans une tension (tout à fait productive) entre pragmatisme éthique d'un côté et réflexion esthétique souveraine de l'autre. Plus précisément : elle vise à une compromission structurelle de l'esthétique par la morale et vice versa, en vue de rehausser leur efficacité réciproque. C'est pourquoi d'ailleurs elle ne saurait mettre en valeur des caractères entièrement intègres : la compromission du narrateur préfigure celle du lecteur qui, quant à lui, est la cible centrale de ces contes, avec tout ce que leur art comporte d'utilitaire. Seulement un lecteur intéressé sera susceptible de s'engager dans une réflexion morale pratique telle qu'elle est envisagée par Diderot, et c'est pourquoi il lui faut des " caractères " au sens fort, c'est-à-dire des personnages littéraires capables d'éveiller et de maintenir l'intérêt. Après la période du drame bourgeois, ce ne sont plus les caractères vertueux, mais les caractères mixtes, ambivalentes qui remplissent cette condition. D'un point de vue pragmatique, ce sont leurs « vices » qui font les « vertus » des personnages de Diderot. Concluons donc que loin d'être le « fondement » de l'éthique Diderotienne, le caractère est son « médium » privilégié, comme le charlatan Desbrosses dans Mystification - un acteur peu fiable mais néanmoins fascinant en tant qu'il miroite entre fiction et pratique, entre la morale et le jeu esthétique.

\section{NOTES}

1. Diderot, D. : Oeuvres complètes, éd. critique par Jean Fabre, Herbert Dieckmann, Jacques Proust et Jean Varloot, Paris, Hermann, 33 vol. prévus, 1975 sqq., Tome XXIV, p. 589. Toutes les références à Diderot dans cette édition (DPV).

2. DPV XXIV, 590. 
3. Titre d'une comédie de Diderot, en quatre actes et en prose, rédigée entre 1775 et sa mort en 1784.

4. Puisque Diderot se plaît à brouiller les limites des genres, il n'est pas toujours facile de savoir quel texte il qualifie comme conte (ou comme dialogue, entretien, etc.). En témoigne l'étonnante variété des éditions. Pour le corpus de cette étude, nous nous tenons à la sélection faite par DPV dans le vol. XII des Oeuvres complètes.

5. Voir p. ex. Ages, A.: " A gift for maxims - Diderot as Moralist. The Testimony of the Correspondence ", New Zealand Journal of French Studies 12/1 (May 1991), p. 5-14. Le fait même qu'une amie de Diderot, Madeleine de Puisieux, publie un livre intitulé Les Caractères en 1750, montre que le genre des caracteres est toujours vivant au siècle des Lumières, et qu'il est pratiqué dans l'entourage le plus proche de Diderot.

6. DPV XII, 72.

7. La Bruyère : « Discours sur Théophraste », Oeuvres complètes, éd. Julien Benda, Paris, 1951, p. 3-18 (p. 5).

8. Cet objectif est aussi au centre de la Satire première de Diderot. Voire DPV XVII, 22 et sqq. pour les exemples.

9. Dans son « Discours sur Théophraste ", La Bruyère écrit qu'il veut « [...] rendre l'homme raisonnable, mais par des voies amples et communes, et en l'examinant indifféremment, sans beaucoup de méthode et selon que les divers chapitres y conduisent, par les âges, les sexes et les conditions, et par les vices, les faibles et le ridicule qui y sont attachés » (op. cit., p. 16).

10. Le philosophe, qui se croyait être un simple observateur, est de plus en plus irrité par les agitations de Rameau. Au souci de la distinction analytique fait désormais écho une confusion qui semble mettre en question tout son être moral (DPV XII, 95) - jusqu'à la fin du dialogue, où il faut tout d'un coup se demander si on est « toujours le même » (DPV XII, 196).

11. Cette opposition entre philosophie morale antique et moderne est thématisée par Anscombe, G.M.E. : « Modern Moral Philosophy », Philosophy 33 (1958), p. 26-42.

12. «La notion morale de caractère [...] joue un rôle essentiel dans la philosophie antique: elle contribue à y rendre cohérente la psychologie de l'action morale, à penser l'unité des vertus au sein d'une personnalité humaine et à englober dans une forme unique les déterminants de l'action qui relèvent de la nature mais aussi de l'éducation et de l'expérience. » Art. " Caractère », Dictionnaire d'éthique et de philosophie morale, publ. sous la dir. de Monique Canto-Sperber, Paris, PUF (2e ed. corr.), 1997, p. 200-209 (p. 200).

13. DPV XXIV, 491. Voir aussi, pour une définition similaire, la p. 494 du même tome.

14. On en retrouve d'ailleurs de multiples traces dans Le Neveu de Rameau, dont le protagoniste se plaint lui-aussi de l'influence néfaste de la « maudite molécule paternelle » (DPV XII, 173).

15. Je renvoie ici à la lecture passionnante de Wilda Anderson (Diderot's Dream, Baltimore, Johns Hopkins Univ. Press, 1990) et aux travaux d'Annie Ibrahim sur le matérialisme de Diderot.

16. Patrick Coleman, qui a étudié la notion de " caractère ", souligne son importance pour le contexte intellectuel du XVIIIe siècle : «I was struck by the ubiquity and resonance of the very term ,caractère' in eighteenth-century discourse. Like ,esprit' or ,moeurs', it is one of those terms whose precise meaning is difficult to pin down but which play a key role in Enlightenment attempt to redefine the shape of man's moral and cultural life. " Coleman, P.: « The Idea of Character in the Encyclopédie ", Eighteenth-Century Studies 13 (1979), p. 21-47, (p. 22 sq). Voir aussi du même auteur : "Character in an Eighteenth-Century Context », The Eighteenth Century, vol. 24, $\mathrm{n}^{\circ} 1,1983$, p. 51-63.

17. Dans le Dictionnaire universel de Furetière (édition de 1707) on peut lire la définition suivante: "Certaine figure qu'on trace sur le papier, sur l'airain, sur le marbre, ou sur autres matières avec la plume, le ciseau, ou autres instruments, pour signifier, ou marquer quelque chose. " La comparaison des articles est intéressante en ce qu'elle met en lumière certaines lacunes de l' Encyclopédie. En particulier, l'article de Encyclopédie omet la définition de « caractère » comme « 
sortilège ", "figure talismanique "- qui, on s'en doute, ne correspond pas à l'intention scientifique de cet ouvrage voué au combat contre le préjugé. Un conte comme Mystification montre pourtant que cette définition est bien présente à l'esprit de Diderot, et qu'il en tire des ressources littéraires remarquables.

18. Je rejoins ici encore une observation faite par Patrick Coleman : «According to this scheme, caractère is a way of talking about things, even when it refers to moral or botanical characters, that is, to qualities that seem rooted in the things themselves. " (" The Idea of Character in the Encyclopédie », op. cit., p. 26.) Selon Coleman, le caractère se range, dans l'Encyclopédie, parmi les « moyens d'expressions "; ce ne serait donc pas seulement une qualité objective (une qualité des objets), mais une « technique d'expression » (ibid., p. 29).

19. L'exposé qui suit est un résumé condensé des idées développées dans mon livre : Diderots Erzählungen. Die Charaktergeschichte als Medium der Aufklärung, Paderborn, Fink, 2014.

20. La présence d'un cadre (et la distinction entre discours et récit qu'il occasionne) n'est pas propre à Diderot, mais appartient à la tradition nouvellistique depuis Boccacce. L'originalité de Diderot consiste plutôt dans l'interruption du cadre, qui n'est plus séparé strictement du récit mais s'y mélange.

21. «Lorsqu'on fait un conte, c'est à quelqu'un qui l'écoute ; et pour peu que le conte dure, il est rare que le conteur ne soit pas interrompu quelquefois par son auditeur. Voilà pourquoi j'ai introduit dans le récit qu'on va lire, et qui n'est pas un conte, ou qui est un mauvais conte, si vous vous en doutez, un personnage qui fasse à peu près le rôle du lecteur, et je commence. » (DPV XII, 521).

22. Le narrateur et son interlocuteur sortent d'une réunion où l'on a passé le temps à lire des contes. Voici comment ils font la critique de ces contes, tout en indiquant les principes poétiques qui gouvernent la production littéraire de Diderot : « [...] un sujet aussi intéressant devrait mettre toutes les têtes en l'air, défrayer pendant un mois tous les cercles de la ville, y être tourné et retourné jusqu'à l'insipidité, fournir à mille disputes, à vingt brochures au moins et à quelques centaines de pièces en vers pour et contre ; et [...] en dépit de toute la finesse, de toutes les connaissances, de tout l'esprit de l'auteur, puisque son ouvrage n'a excité aucune fermentation violente, il est médiocre et très médiocre» (DPV XII, 521).

23. Voir la note 21 . Méchant et mauvais étant, dans la langue du temps, des synonymes (« méchant auteur »).

24. « La traîtresse !» (DPV XII, 524).

25. DPV XII, 534 sq.

26. DPV XII, 550.

27. DPV XII, 568.

28. Il en va ici de la différence entre la personne et le personnage. Comme l'a très justement observé David Oakleaf, le « caractère » au XVIIIe siècle est, toujours, en partie, constitué par la réputation : "Character is a publicly circulated sign. " Oakleaf, D. : " Marks, Stamps and Representations: Character in Eighteenth-Century Fiction ", Studies in the Novel, vol. 23, n³ (1991/Fall), p. 295-311 (p. 299).

29. « C'est qu'on ignore ces choses-là. C'est qu'il faut se taire quand on ignore » (DPV XII, 552).

30. Tel est le sens du dénouement alternatif proposé à la fin. L'interlocuteur montre qu'il a bien compris sa leçon, quand il dit : «Je change la thèse, en supposant un procédé plus ordinaire à Mme de la Carliere » (DPV XII, 573).

31. «Et puis j'ai mes idées, peut-être justes, à coup sûr bizarres, sur certaines actions que je regarde moins comme des vices de l'homme que comme des conséquences de nos législations absurdes, sources de moeurs aussi absurdes qu'elles et d'une dépravation que j'appellerais volontiers artificielle. Cela n'est pas trop clair, mais cela s'éclaircira peut-être une autre fois... » (DPV XII, 575). 
32. Diderot parle d'une traduction, qui est aussi une trahison : «Et c'est ainsi que de bouche en bouche, échos ridicules les unes des autres, un galant homme est traduit pour un plat homme, un homme d'esprit pour un sot, un homme honnête pour un coquin, un homme de courage pour un insensé, et réciproquement » (DPV XII, 551).

33. Tels p.ex. la visibilité de la personne sur la scène publique, sa position dans la hiérarchie sociale etc.

34. DPV XII, 546 sq.

35. Notons en passant que le lecteur se voit ainsi assigner la place qu'occupait ci-devant le pouvoir transcendant!

36. Le même procédé se trouve à la fin de Madame de La Carlière. Le narrateur demande à son interlocuteur : « Mais si vous aviez une fille à marier, la donneriez-vous à Desroches ? » (DPV XII, 574). Encore une fois, l'acquittement théorique du mari infidèle est suivi d'un jugement plus sévère au plan de la pratique.

37. Dans Madame de La Carlière, le temps est évoqué comme facteur non seulement du développement des caractères, mais aussi de celui de l'opinion publique (DPV XII, 572).

38. C'est ce que montre, entre autres, le régime des voiles restreignant la licence sexuelle et le plaisir érotique des Otaïtiens (DPV XII, 618 sq.).

39. Le personnage « $\mathrm{A}$ » propose à son interlocuteur: «Interrogeons bonnement la nature, et voyons sans partialité ce qu'elle nous répondra sur ce point » (DPV XII, 630 sq.). Suit une discussion sur le mariage, la galanterie, la coquetterie et la jalousie qui montre que tous ces phénomènes ne sont pas de simples artifices de la vie civilisée, mais existent bel et bien à l'état de nature.

40. Ce terme doit son essor aux travaux de Michel Foucault. Voire Foucault, M. : « À propos de la généalogie de l'éthique : un aperçu du travail en cours », Dits et Ecrits : 1954-1988, éd. établie sous la dir. de Daniel Defert et François Ewald, avec la collaboration de Jacques Lagrange, Tome IV, Paris, Gallimard, 1994, p. 609-631.

41. DPV XX, 50-51.

42. Selon Annie Ibrahim, Diderot tenterait de surmonter, dans le cadre de son matérialisme, " l'alternative entre analytique de la forme et poétique de la force ». Bien que je ne suive pas ici le côté aléatoire de sa lecture, je pense qu'elle a raison en supposant que c'est " à une recomposition de la notion du Soi que travaille Diderot ». C'est précisément dans ce contexte-là qu'il faut voir, à mon sens, les variations de Diderot sur le thème du caractère. Ibrahim, A. : « Le matérialisme de Diderot : formes et forces dans l'ordre des vivants ", ds : Diderot et la question de la forme, coordonné par A. Ibrahim, Paris, Presses Universitaires de France, 1999, p. 87-103 (p. 93).

43. DPV XXIII, 171.

44. Le rapprochement sémantique entre le sort et les sentiments a été fait par Diderot dès Ceci n'est pas un conte, où il traite la passion comme une fatalité, c'est-à-dire, dénuée de toute moralité. Voire DPV XII, 533.

45. Benjamin, W. : "Schicksal und Charakter ", ds: Illuminationen. Ausgewählte Schriften, éd. Siegfried Unseld, Frankfurt a. M., Suhrkamp, 1961, p. 47-55.

46. Philippe Lacoue-Labarthe a fait la même observation en parlant, à propos du jeu de l'acteur, de « mimésis [...] active ». « Diderot, le paradoxe et la mimésis », Poétique XI (1980), p. 267-281 (p. 279).

47. Comme le souligne Colas Duflo (Diderot : du matérialisme à la politique, Paris, CNRS Ed. 2013, chap. IV), la notion de « volonté » (comme d'ailleurs celle de « liberté »), est pour Diderot un « mot vide de sens ». Cela ne l'empêche cependant pas de l'intégrer à son concept du personnage littéraire, en le transférant comme ici dans un cadre quasi-mythologique.

48. Pour les paroles exactes du Marquis, voir DPV XXIII, 168.

49. DPV XII, 546. Cela devient plus clair encore quelque lignes après, quand il s'agit de tirer une conclusion des deux histoires qui forment le sujet du conte : "Mais cela est encore à peu près 
dans la règle. S'il y a un bon et honnête Tanié, c'est à une Reymer que la Providence l'envoie. S'il y a une bonne et honnête de La Chaux, elle deviendra le partage d'un Gardeil, afin que tout soit fait pour le mieux. » (Je souligne).

50. DPV XII, 453.

51. Les différents niveaux de la communication ironique sont analysés par Ruth Groh dans Ironie und Moral im Werk Diderots, München, Wilhelm Fink, 1984 (p. 22 sqq.).

52. Selon la terminologie de Wolfgang Iser (Der Akt des Lesens. Theorie ästhetischer Wirkung, 1976).

53. Voir à ce propos les analyses toujours actuelles de Kempf, R. : Diderot et le roman ou le démon de la présence, Paris, éd. du Seuil, 1964, p. 191-235.

54. DPV XII, 493.

55. Dans la Satire Première, Diderot exprime exactement la même idée : «Heureuse la société où chacun serait à sa chose et ne serait qu'à sa chose !» (DPV XII, 20). On peut tracer les origines de cette Conception de la cité juste, connue aujourd'hui sous le titre d'« idiopragie ", jusqu'au Politique de Platon.

56. « [...] ce ne sont plus, à proprement parler, les caractères qu'il faut mettre sur la scène, mais les conditions. Jusqu'à présent, dans la comédie le caractère a été l'objet principal, et la condition n'a été que l'accessoire ; il faut que la condition devienne aujourd'hui l'objet principal, et que le caractère ne soit que l'accessoire. [...] C'est la condition, ses devoirs, ses avantages, ses embarras qui doivent servir de base à l'ouvrage. Il me semble que cette source est plus féconde, plus étendue, et plus utile que celle des caractères » (DPV X, 144). Cette dévaluation, souvent citée, du « caractère » par Diderot est probablement une des raisons pour lesquelles la fonction vraiment essentielle de ce concept dans ses textes de prose a largement échappé à l'attention des critiques. 57. DPV X, 144.

58. Cela montre à quel point le concept de « caractère » a évolué depuis l'âge classique. Voire à ce sujet: Jauss , H.-R. : « Vom plurale tantum der Charaktere zum singulare tantum des Individuums », ds: Individualität, publ. sous la dir. de Manfred Frank, München, Wilhelm Fink, 1988, p. 237-269.

59. DPV XII, 455.

60. DPV XII, 452.

61. DPV XII, 442.

62. Voilà comment, à mon avis, il faut lire « l'exemple emprunté d'un autre art » dans la poétique du conte historique (DPV XII, 455). La cicatrice appliquée par le peintre sur la tête idéale montre qu'il ne saurait y avoir d'idéalité entièrement intègre. L'idéal est délibérément " corrompu ", pour le rendre plus proche de la réalité.

63. Edmiston, W. F. : "The Role of the Listener. Narrative Technique in Ceci n'est pas un conte ", Diderot Studies 20 (1981), p. 61-75 (p. 62).

64. «On me demandera si je n'ai jamais ni trahi, ni trompé, ni délaissé aucune femme sans sujet » (DPV XII, 546).

65. Mademoiselle Dornet appelle Diderot « notre esprit fort » (DPV XII, 398).

66. D'ailleurs, de nombreux éléments aident à mettre en valeur le caractère dramatique de ce texte, comme par exemple les parties dialoguées et certains commentaires méta-fictifs du narrateur (DPV XII, 390 sq.).

67. DPV XII, 455. Diderot, comme Desbrosses, fait « danser » Mademoiselle Dornet, en l'irritant par ses réponses.

68. DPV XII, 397. 


\section{RÉSUMÉS}

Cet article tente de démontrer la cohérence des contes de Diderot envisagés sous le point de vue d'un programme étho-poétique englobant, au centre duquel se trouve la notion de caractère. Le caractère chez Diderot semble donner un fondement quasi physique, en tout cas naturaliste à la morale, mais il est lui-même miné par des tensions (entre forme et force, être et paraitre, fiction et morale etc.) que met en relief l'article. Après une première réflexion sur la critique du jugement moral effectuée par les contes, qui permet de relever les 'vertus' du caractere du point de vue de la pensée morale, s'approfondit l'analyse des enjeux littéraires des contes. Il s'avère que plutôt qu'un fondement stable de la morale, le caractère (littéraire), grâce à ses ambivalences mêmes, constitue le médium privilégié de la stratégie esthétique de Diderot en tant qu'elle vise essentiellement à une compromission éthique du lecteur.

This article proposes to demonstrate the coherence of Diderot's tales from the point of view of an overarching ethico-poetic strategy, centered on the notion of "character". Character seems to provide Diderot's moral thought with a quasi-physical, in any case naturalistic basis - were it not for the internal tensions with which it is ridden (between form and force, essence and appearance, fiction and morality etc.) and which this article tries to bring to the fore. Whereas a first section deals with the critique of moral judgment in and by the tales, thus allowing to highlight the 'virtues' of the notion of character from the point of view of ethical thinking, the latter part is devoted to investigating the properly narrative stakes of the tales. It concludes that, rather than providing any kind of stable "foundation" to his ethical system, the notion of (literary) character, thanks to its very ambivalence, constitutes a pivotal element of Diderot's aesthetic strategy whose aim is the involvement - and ultimate corruption - of the reader.

\section{INDEX}

Keywords : Character, anthropology, history, critique, judgment

Mots-clés : Caractere, anthropologie, histoire, critique, jugement

\section{AUTEUR}

\section{KONSTANZE BARON}

Eberhard Karls Universität Tübingen, Allemagne. konstanze.baron@unituebingen.de /

kbaron@fas.harvard.edu

Elle est enseignante-chercheuse au grade de Akademische Rätin au Séminaire de Langues et Littératures Romanes de l'Université de Tuebingen et, pendant l'année 2015-2016, détentrice d'un postdoctoral fellowship au Mahindra Humanities Center à Harvard. Après des études de lettres modernes à l'Université d'Oxford, elle a obtenu son DEA en « Histoire et Sémiologie du texte » à Paris VII en 2003. De 2009 à 2015, elle a été attachée de recherche au Centre pour les Recherches sur les Lumières en Europe (IZEA) à Halle. Sa thèse de doctorat, soutenue à l'Université de 
Konstanz en 2010 et publiée en 2014 par Fink Verlag sous le titre «Diderots Erzählungen. Die Charaktergeschichte als Medium der Aufklärung ", explore la dimension éthopoétique des contes de Denis Diderot. D'autres travaux sur Diderot, Rousseau et la philosophie morale des Lumières sont actuellement en cours de parution.

She is assistant professor (Akademische Rätin) at the Department of Romance Literatures and Languages in Tuebingen, and, during the academic year 2015-16, a postdoctoral fellow at the Mahindra Humanities Center at Harvard. She studied Modern French and History in Oxford and in 2003 obtained her DEA in "Histoire et Sémiologie du texte" from Paris VII. From 2009 to 2015, she was a full-time researcher at the Center for European Enlightenment Studies (IZEA) in Halle. Her dissertation, which she defended in 2010 at the University of Konstanz and which was published in 2014 by Fink Verlag under the title "Diderots Erzählungen. Die Charaktergeschichte als Medium der Aufklärung“, investigates the ethico-poetic dimension of Diderot's tales. Other current projects are dealing with the theory of action/acting, the role of "Enlightenment Genius" and of institutions in Rousseau. 